\title{
Analysis of Doppler Effect in OFDM signals for Underwater Acoustic Channels
}

\author{
Ranjani.G ${ }^{\# 1}$, Sadashivappa.G ${ }^{* 2}$

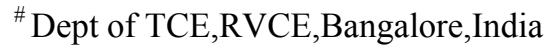 \\ ${ }^{1}$ ranjanig@rvce.edu.in \\ ${ }^{*}$ Dept of TCE,RVCE,Bangalore,India \\ ${ }^{2}$ sadashivappag@rvce.edu.in
}

\begin{abstract}
Acoustic communication is best supported at low frequency. Since the available bandwidth is less, higher order modulation techniques such as OFDM are employed. Inherent motion of the system poses stringent limitation on the synchronization issues. Thus modelling of multipath propagation especially Doppler Effect plays an important role in the efficient design of high data rate acoustic communication systems. Since OFDM is very sensitive to carrier frequency offsets, Doppler correction techniques need to be developed to mitigate the severity of Inter Carrier Interference (ICI).This paper presents the development of an OFDM based underwater acoustic communication system and analyses its performance.
\end{abstract}

Keyword- OFDM, underwater communication, Acoustic communication, Doppler Effect, Channel modelling

\section{INTRODUCTION}

Bandwidth efficiency is proportional to the guard interval between OFDM blocks and the number of carriers. As the number of carriers increases in a given bandwidth, both the bandwidth efficiency and the coherence between carriers increase however the length of the OFDM block increases, reducing the coherence time of the channel. In such a case ICI dominates the detection error. Several approaches for mitigation of Doppler with coherent detection is available in the literature

Srinivas Yerramalli and Urbashi Mitra [1] proposed optimal resampling of OFDM signals for multiscale multilag underwater acoustic channels. This work examines the influence of resampling parameter in a multiscale scenario by matched filtering the incoming signal with an approximated channel function on each path. Zhaohui Wang, Shengli Zhou, and Lei Wan Xiaoka Xu, proposes a parameterization technique for both path amplitude and delay variations of underwater acoustic channels for block decoding of orthogonal frequency division multiplexing,[2]. This paper proposes a channel parameterization using one polynomial to approximate the amplitude variation and another polynomial up to the first order to approximate the delay variation within block duration.

Kai Tu, Tolga M. Duman, Milica Stojanovic, and John G. Proakis proposed multiple-resampling receiver design for OFDM over doppler-distorted underwater acoustic channels, [3] This paper, focuses on orthogonal frequency-division multiplexing (OFDM) receiver designs for underwater acoustic (UWA) channels with Doppler distortions. The paper points out that though the resampling process improves the performance, it results in the significant residual Doppler distortion; causing severe degradation in the system performance. It is demonstrated that compensation of Doppler distortion improves BER performance and ICI power reduction.

Bayan S. Sharif et al [4] proposed a Doppler compensation approach that provides a a Doppler estimation technique and sample rate conversion to remove Doppler shift induced by platform velocity and acceleration. Limin $\mathrm{Yu}$, and L.B. White, paper proposed a broadband Doppler compensation structure based on rational orthogonal wavelet signaling and perfect reconstruction wavelet filter banks [5]. Baosheng Li et al proposed a two-step approach to mitigate the Non uniform Doppler Shifts [6] In this paper, the channel is treated to have a common Doppler scaling factor on all propagation paths, The receiver is based on block-by-block processing, and does not rely on channel dependence across OFDM blocks. The proposed practical receiver algorithms rely on the preamble and post amble of a packet consisting of multiple OFDM blocks to estimate the resampling factor, the null subcarriers to facilitate high-resolution residual Doppler compensation, and the pilot subcarriers for channel estimation.

A.E. Abdelkareem et al have proposed a time varying Doppler-shift compensation scheme for wideband orthogonal frequency division multiplexing (OFDM) suitable for shallow water communications [7]. This scheme accommodates a channel model having velocity that accelerate and de-accelerate during a packet of multiple OFDM frames. To deal with this variation, the time expansion/compression is measured within a fraction of the sample period and then compensated using sample-by-sample interpolation. The Doppler-shift and its residual are frequently estimated during the symbol time by estimating the first order moment of the 
cyclic prefix correlation and then compensating the Doppler via an efficient 4th order Lagrange interpolation.In the proposed method, it is assumed that the channel variation is mainly caused by the motion of both transmitter and receiver, leading to a significant time varying Doppler-shift. The Doppler-shift is assumed variable during the symbol time, however, it is assumed constant in all paths. Gang Qiao et al have deliberated Doppler estimation and the respective compensation technique along with various diversity techniques for OFDM-based systems [8]. Frequency domain re-sampling (FDR) is introduced in this paper, and the Fast FDR (FFDR) method is also provided to carry out the Doppler shift compensation more efficiently.M. Stojanovic, J. Catipovic and J. G. Proakis have proposed an adaptive multichannel combining and equalization technique for underwater acoustic communications [9]. The receiver structure used is based on the optimal multichannel DFE, an RLS based algorithm for fast tracking capabilities.

\section{SYSTEM MODEL}

Understanding the double dispersive nature of the channel with limited bandwidth, OFDM with QPSK is selected as a modulation technique. To design the OFDM system, Bandwidth and Carrier frequency need to be assigned. Carrier frequency spacing, symbol rate should be identified based on the Doppler shift.

Identification of Carrier frequency and Bandwidth is based on Attenuation or path loss that occurs in an underwater acoustic channel. It varies with range 1 and a signal of frequency $f$ and is given by

$$
A(l, f)=A_{0} l^{k} a(f)^{l}
$$

where $A_{0}$ is a unit-normalizing constant, $k$ is the spreading factor, and $a(f)$ is the absorption coefficient. The ambient noise in the ocean varies with turbulence, shipping, waves, and thermal noise. The overall p.s.d. of the ambient noise is given by

$$
N(f)=N_{t}(f)+N_{s}(f)+N_{w}(f) N_{t h}(f)
$$

Where $\mathrm{N}_{t}(\mathrm{f})$ is the p.s.d of the turbulence noise $\mathrm{N}_{\mathrm{s}}(\mathrm{f})$ is the p.s.d of the ship noise $\mathrm{N}_{w}(f)$ is the p.s.d of the wind noise $\mathrm{N}_{\mathrm{th}}(\mathrm{f})$ is the p.s.d of the thermal noise.The p.s.d. of the ambient noise, $\mathrm{N}(\mathrm{f})$ in $\mathrm{dB}$ re $\mu$ Pa per $\mathrm{Hz}$ is a function of frequency. For a moderate shipping activity $(\mathrm{s}=0.5)$ and wind speed of $10 \mathrm{~m} / \mathrm{s}$.

System bandwidth may be defined as the range of frequencies around Optimal frequency $\mathrm{f}_{\mathrm{o}}(1)$ for which

$$
\operatorname{SNR}(l, f)>\operatorname{SNR}\left(l, f_{0}(l)\right) / 2
$$

If equal energy distribution across the $3 \mathrm{~dB}$ bandwidth is assumed with threshold SNR to be $\mathrm{SNR}_{0}$ then the transmitted power and the capacity is given respectively by

$$
\begin{gathered}
P_{3}(l)=S N R_{0} B_{3}(l) \frac{\int \frac{B_{3}(l)}{\int} N(f) \cdot d f}{\int_{B_{3}(l)}^{-1}(l, f) \cdot d f} \\
C_{3}(l)=\int_{B_{3}(l)} \log _{2}\left[1+\frac{P_{3}(l) / B_{3}(l)}{A(l, f) N(f)}\right]
\end{gathered}
$$

Range is chosen such that it supports telemetry and networking applications. Keeping the power transmitted as minimum power, the symmetric $3-\mathrm{dB}$ bandwidth obtained is $10.8 \mathrm{KHz}$. and the carrier frequency is $9.2 \mathrm{KHz}$ as shown in Table I

Table I

Choice of Carrier Frequency

\begin{tabular}{|l|l|}
\hline \multicolumn{2}{|l|}{ Range=5Km; k=1.5; p=0.3MW } \\
\hline $\mathbf{f ~ ( H z )}$ & SNR (dB) \\
\hline $3.8 \mathrm{~K}$ & 22 \\
\hline $9.2 \mathrm{~K}$ & 25 \\
\hline $14.6 \mathrm{~K}$ & 23 \\
\hline
\end{tabular}

\section{OFDM TRANSMITTER}

With the calculated bandwidth and carrier frequency, the OFDM parameters are derived and tabulated in Table II. OFDM symbols can be generated through IDFT and can be represented as

$$
S_{n}=S(n T)=\frac{1}{\sqrt{N}} \sum_{k=0}^{N-1} I_{k} e^{\frac{j 2 \pi m k}{N}}
$$

Where $\mathrm{I}_{\mathrm{k}}$ refers to the QPSK modulated symbol to be transmitted in the $\mathrm{k}^{\text {th }}$ subcarrier while $\mathrm{N}$ is the total number of subcarriers considered. 
Table II

OFDM Parameters

\begin{tabular}{|c|c|c|}
\hline Parameter & Formula & values \\
\hline Range $(\mathrm{Km})$ & & 5 \\
\hline SNR $(\mathrm{dB})$ & & 22 \\
\hline Sound speed $(\mathrm{m} / \mathrm{s})$ & & 1500 \\
\hline fc $(\mathrm{KHz})$ & & 9.2 \\
\hline Band width (KHz) & & 10.8 \\
\hline $\mathrm{f}_{\mathrm{L}}(\mathrm{KHZ})$ & $f_{c}-\frac{B}{2}$ & 3.8 \\
\hline $\mathrm{f}_{\mathrm{H}}(\mathrm{KHZ})$ & $f_{c}+\frac{B}{2}$ & 14.7 \\
\hline $\mathrm{f}_{\mathrm{S}}(\mathrm{KHZ})$ & $F_{S} \gg 2 f_{H}$ & 100 \\
\hline Guard Intervel (ms) & $T_{g} \geq \tau_{\max }$ & 45 \\
\hline No: of Zero padding & & 4500 \\
\hline No: of carriers & $\mathrm{N}$ & 1024 \\
\hline Carrier spacing (HZ) & $\Delta f=\frac{B}{N}$ & 10.55 \\
\hline Symbol duration (ms) & $T_{S C}=T_{d}=\frac{1}{\Delta f}$ & 94.8 \\
\hline OFDM length & & 9480 \\
\hline OFDM symbol duration $(\mathrm{ms})$ & $T_{\text {sym }}=T_{d}+T_{g}$ & 139.8 \\
\hline Modulation & & QPSK \\
\hline No:Pilots & & 120 \\
\hline Blocks per frame & & 7 \\
\hline Frame duration $(\mathrm{ms})$ & $($ Blocks/Frame $) *($ Symbol duration $)$ & 980 \\
\hline Frames per transmission & & 1 \\
\hline Coherence bandwidth $(\mathrm{Hz})$ & $B_{C} \approx \frac{1}{5 \tau_{\max }}$ & 4.4 \\
\hline Vehicular motion(m/s) & & 1 \\
\hline Doppler shift(Hz) & $f_{d}=f_{c} \frac{v}{c}$ & 6.13 \\
\hline Coherence Time(ms) & $T_{C}=\sqrt{\frac{9}{16 \pi \mathrm{f}_{\mathrm{d}}^{2}}}$ & 69 \\
\hline Bandwidth efficiency & $\eta=\frac{R}{B}$ & 2 \\
\hline
\end{tabular}

The signal gets corrupted with Inter Symbol Interference (ISI) and Inter Carrier Interference (ICI) as it passes through a multipath channel. To overcome these problems, guard intervals have to be added. Guard intervals can be created by adding cyclic prefix or Zero padding. The main advantage of using zero padding is that no extra power is required for transmission. The zero padded signals is given by

$$
g(t)=\left\{\begin{array}{lc}
1 & t \in[0, T] \\
0 & t \in\left[T, T+T_{g}\right.
\end{array}\right]
$$

where $\Delta f$ is the subcarrier spacing and Zero padding is represented as

$$
S(t)=\frac{1}{\sqrt{N}} \sum_{k=0}^{N-1} I_{k} e^{j 2 \pi k \Delta t t} g(t) ; t \in\left[0, T+T_{g}\right]
$$

Thus the transmitted signal (frame) can be written as

$$
S(t)=\operatorname{Re}\left[\frac{1}{\sqrt{N}}\left[\sum_{k=0}^{N-1} I_{k} e^{j 2 \pi k \Delta f t} g(t)\right] e^{j 2 \pi f_{c} t}\right] ; t \in\left[0, T+T_{g}\right]
$$

The transmitted frame is shown in fig2.Synchronization of the transmitter and the receiver in both time and frequency is essential for OFDM to reduce the frequency selectivity of the channel. Poor timing synchronization results in symbol timing offset which leads to a phase offset in the frequency domain. Poor frequency synchronization results in Phase noise, carrier frequency offset. Carrier frequency offset results in ICI. In this paper Synchronization through pilot tones that are employed in each block of OFDM is considered. 


\section{CHARACTERIZATION OF THE UA CHANNELS}

In general channel can be simulated with models such as ray tracing and parabolic expansion models. The output is a channel response that incorporates time variability. With this initial setup In this paper Channel simulations are based on [10] is considered. The channel can be expressed mathematically

$$
h(t)=\sum_{p} h\left(t-\tau_{p}\right)
$$

where $\tau_{p}=\frac{l_{p}}{c}$ is the path delay; $l_{p}$ is the path length. The path delay, $\tau_{p}(t)$ need to be modelled. This depends on the range, velocity of sound and relative velocity. This can be estimated by sounding techniques.

In a multipath channel, all the paths will have a different gain and delay. The frequency and reflection coefficients determine the total number of paths. The variation in path gain and delay are termed as large scale variations. Additional variations appear due to surface scattering and movement of transmitters and receivers. The overall transfer function of the Multipath channel is given by

$$
H(f, t)=H_{0}(f) \sum_{p i} h_{p, i}\left(e^{j 2 \pi f\left(\tau_{p}+\delta \tau_{p, i}\right)}\right)\left(e^{j 2 \pi a} p f t\right)
$$

Where $\mathrm{H}_{0}(\mathrm{f})$ is the reference path gain, $\mathrm{h}_{\mathrm{p}, \mathrm{i}}$ is the Gain of the $\mathrm{p}^{\text {th }}$ path given by

$$
\mathrm{h}_{\mathrm{p}, \mathrm{i}}=\frac{\Gamma_{\mathrm{p}}}{\sqrt{\mathrm{A}\left(1_{\mathrm{p}}, \mathrm{f}\right)}}
$$

$\Gamma_{\mathrm{p}}$ is the cumulative reflection coefficient along $\mathrm{p}^{\text {th }}$ path, $\mathrm{A}\left(\mathrm{l}_{\mathrm{p}}, \mathrm{f}\right)$ is the propagation loss along the path. $\tau_{\mathrm{p}}$ is the path delay, $a_{p}=f_{c} \frac{v}{c} \cos \theta$ is the Doppler coefficient along the path and $\delta \tau_{\mathrm{p}, \mathrm{i}}$ is the delay due to small scale variation. Due to local variations, a single path itself divides further into several intra paths, each with a different Doppler shift that contributes delay due to small scale variation. The Doppler shift in each path has three components namely variation due to drift, vehicular and surface. They are calculated as

$$
\begin{aligned}
& a_{d r}=\frac{v_{d r}}{c} \quad a_{v l}=\frac{v_{v l}}{c} \quad a_{s u r}=\frac{v_{\text {sur }}}{c} \\
& v_{d r}=v_{t d} \cos \left(\theta_{p}-\theta_{t d}\right)-v_{r d} \cos \left(\theta_{p}+\theta_{r d}\right) \\
& v_{v l}=v_{t v} \cos \left(\theta_{p}-\theta_{t v}\right)-v_{r v} \cos \left(\theta_{p}+\theta_{r v}\right)-\left(v_{t v} \cos \left(\theta_{0}-\theta_{t v}\right)-v_{r v} \cos \left(\theta_{o}+\theta_{r v}\right)\right) \\
& v_{\text {sur }}=2 v_{w} \sin \left(\theta_{p}\right)\left(\sum_{p} \sin \left(2 \pi k n_{s p}-\pi\right)+2 \pi f_{w} t\right)
\end{aligned}
$$

and the effective Doppler shift is given by

$$
a_{\text {eff }}=a_{0}+\sum_{p} a_{p}
$$

where $\mathrm{a}_{0}=0$ is the initial Doppler shift parameter and $a_{p}=a_{d r}+a_{v l}+a_{\text {sur }}$ is the Doppler shift occurred in 'p'th path

\section{RECEIVER ALGORITHM}

The OFDM signal is corrupted by noise and multipath effects as it passes through the channel. The received signal can be written as

$$
y(t)=\sum_{k=0}^{N-1}\left\{I_{k} e^{j 2 \pi k \Delta t} e^{j 2 \pi a f_{k} t}\left[\sum_{p} e^{j 2 \pi f_{k} \tau_{p}} g\left(t+a t-\tau_{p}\right)\right]\right\}+w(t)
$$

where $\tau_{p}$ is the path delay and a is the effective Doppler function $\mathrm{H}(\mathrm{f})$ at $\mathrm{k}^{\text {th }}$ carrier frequency and $\mathrm{w}(\mathrm{t})$ is the white noise. The received signal is down converted, zero padding is removed and the OFDM symbols are demodulated through FFT and the Doppler compensation is done through carrier sliding. The outputs of the FFTs form the decision variables given by

$$
\mathrm{y}_{\mathrm{k}}=\sum_{\mathrm{l}=0}^{\mathrm{K}-1} R_{\mathrm{y}}(k, l) \mathrm{I}_{\mathrm{k}}+w_{k}
$$




$$
R_{y}(k, l)=\int_{0}^{T} H(t) e^{j 2 \pi(l-k) \Delta t t} d t
$$

However the bit error rate obtained is high of the order of $10^{-1}$. This result directs to move further in Identifying Possible Equalization Methods to compensate for ISI that dominates the degradation. Linear MMSE equalization can be defined by

$$
\begin{aligned}
& \hat{I}_{k}=Q_{y}^{H} Y \text { where } \\
& Q_{y}=\left(R_{y} R_{y}^{H}+2 N_{0} T I\right)^{-1} R_{y}
\end{aligned}
$$

And the mean square error is calculated by

$$
M S E=1-\frac{\operatorname{tr}\left[R_{y}^{H} Q_{y}\right]}{K}
$$

\section{SIMULATION RESULTS}

The OFDM system model is shown in Fig1, The channel is simulated with various values of depths in which the receiver is placed and corresponding arrivals are calculated. The parameters considered for simulation along with the obtained number of arrivals is listed in Table III.

Table III

Number of arrivals

\begin{tabular}{|l|l|l|l|}
\hline Range(Km) & 1 & 1 & 1 \\
\hline Sound speed(m/s) & 1496 & 1496 & 1496 \\
\hline Carrier frequency $(\mathrm{Hz})$ & $10 \mathrm{~K}$ & $10 \mathrm{~K}$ & $10 \mathrm{~K}$ \\
\hline Signal frequency(Hz) & $3 \mathrm{~K}$ & $3 \mathrm{~K}$ & $3 \mathrm{~K}$ \\
\hline Depth(m) & 2 & 5 & 8 \\
\hline Number of arrivals & 27 & 31 & 33 \\
\hline
\end{tabular}

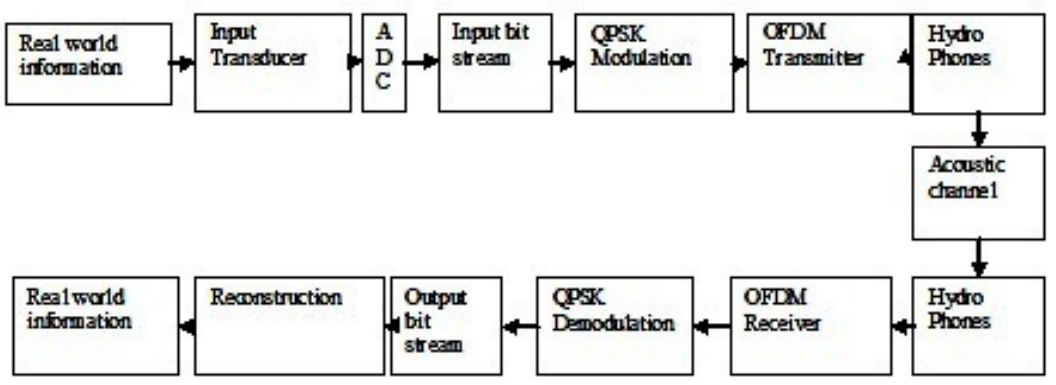

Figure 1 System Model

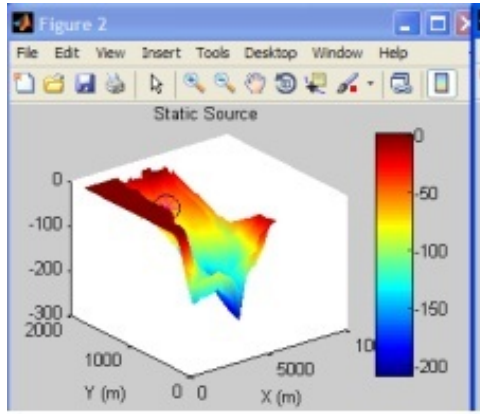

(a)

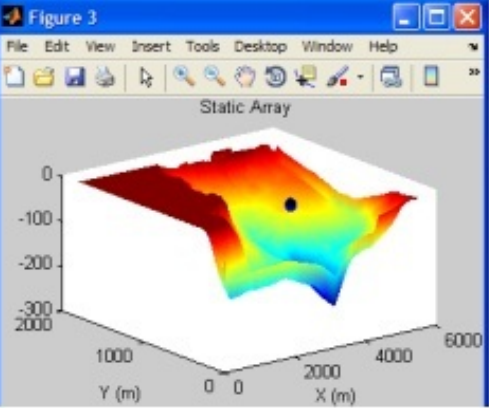

(b)

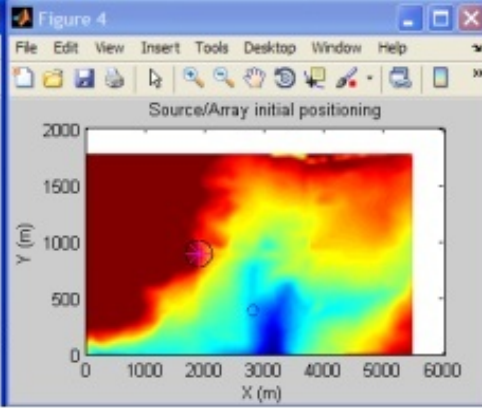

(c)

Figure 2 Transmitter and receiver positions (a) source position (b) receiver array position(c) source-receiver positioning for static source and receiver

The position of source and receiver is shown in fig2. The impulse response and its spectrum in time frequency and delay frequency units for depth of $2 \mathrm{~m}, 5 \mathrm{~m}$, and $8 \mathrm{~m}$ are calculated with the channel model equations described in section IV and are plotted respectively in fig $3,4,5$. 


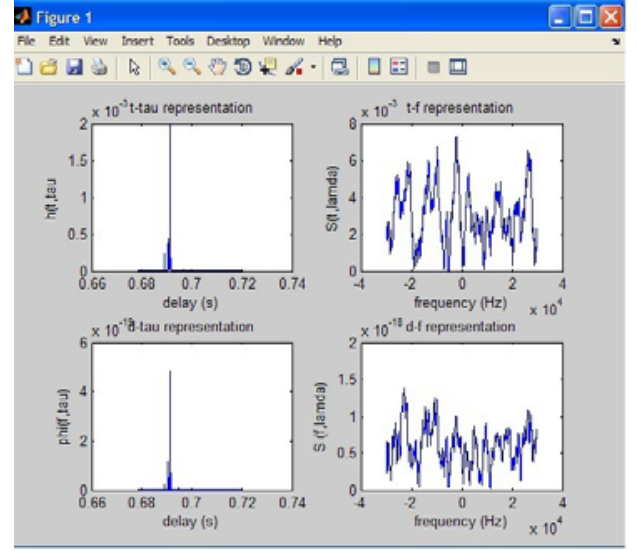

Figure 3 Impulse responses and its spectrum for receiver at $2 \mathrm{~m}$ depth;Top Left: impulse response in time and delay units; Bottom Left: impulse response in delay and frequency units;Top Right: spectrum of impulse response in time and delay frequency units; Bottom Right: spectrum of impulse response in frequency and delay frequency units

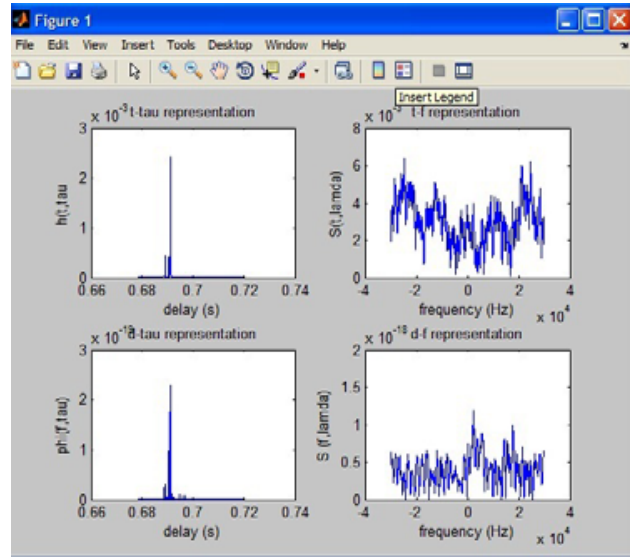

Figure 4 impulse responses and its spectrum for receiver at depth $5 \mathrm{~m}$;Top Left: impulse response in time and delay units; Bottom Left: impulse response in delay and time frequency units; Top Right: spectrum of impulse response in time and delay frequency units; Bottom Right: spectrum of impulse response in time frequency and delay frequency units

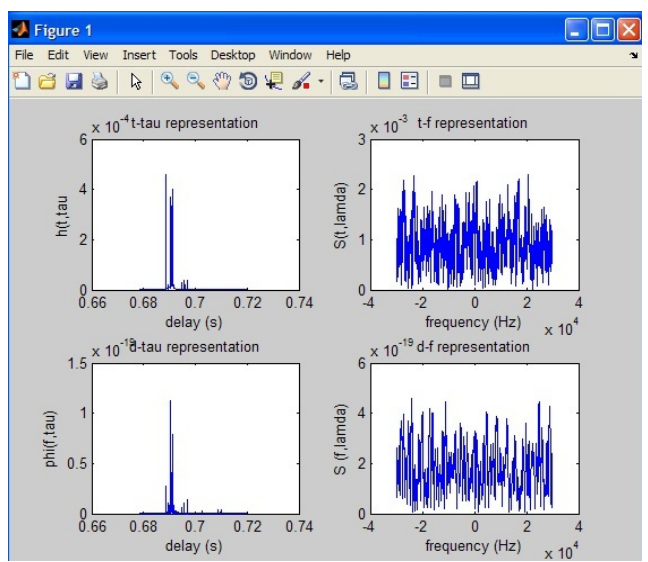

Figure 5 impulse responses and its spectrum for receiver at depth $8 \mathrm{~m}$; Top Left: impulse response in time and delay units; Bottom Left: impulse response in delay and time frequency units; Top Right: spectrum of impulse response in time and delay frequency units; Bottom Right: spectrum of impulse response in time frequency and delay frequency units. 


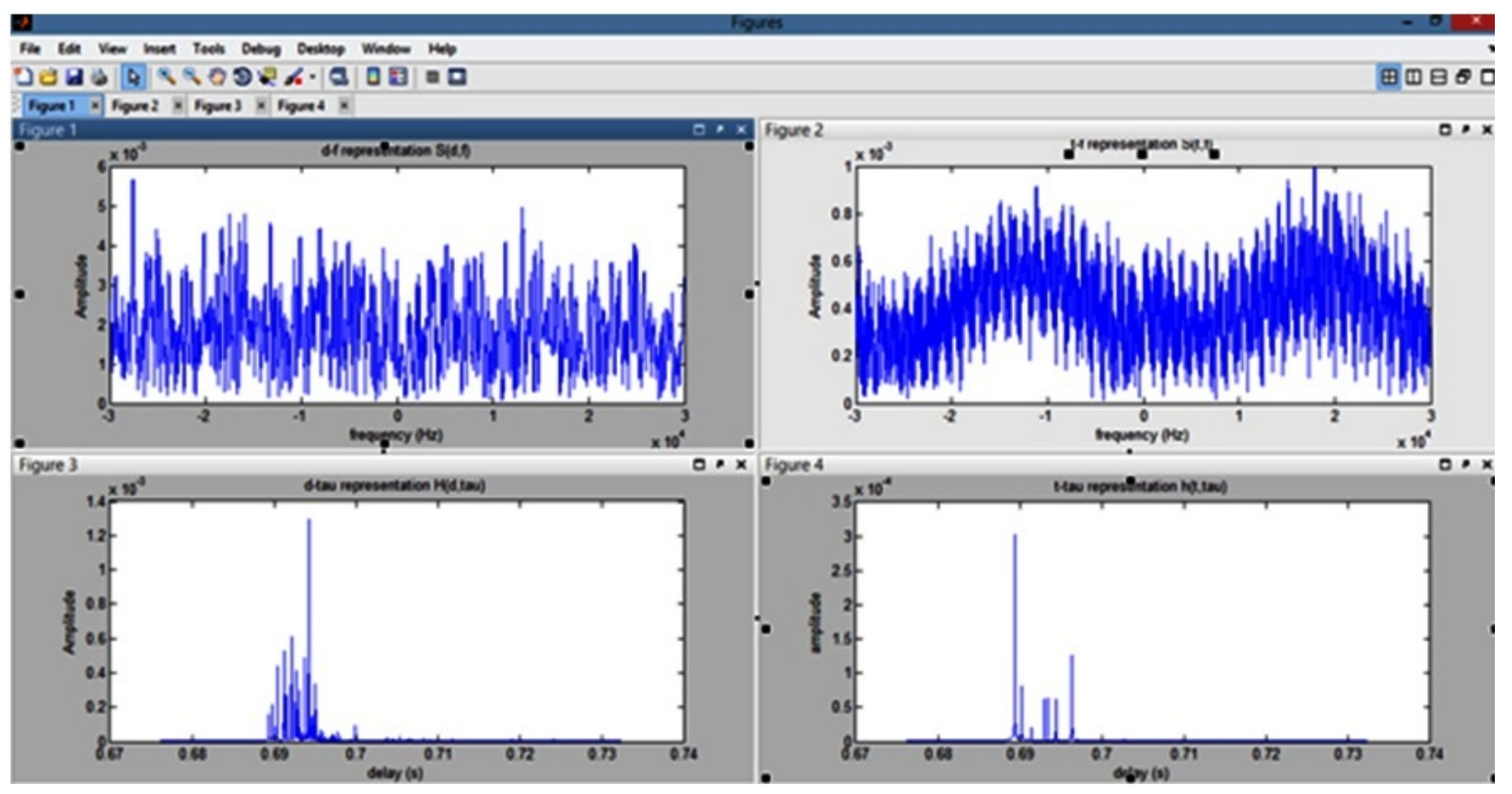

Figure 6 Impulse responses and its spectrum for frequency selective fast fading channel; Top Left: spectrum of impulse response in time frequency and delay frequency units; Top Right: spectrum of impulse response in time and delay frequency units; Bottom Left: impulse response in delay and time frequency units; Bottom Right: impulse response in time and delay units;

From the results, it is observed that when the receiver is placed above the source depth, many of the arrived rays destructively interfere with each other resulting in lesser number of arrivals at the receiver point. Thus the impulse response functions are loosely packed and as depth increases to source depth level and more, the interference becomes lesser and lesser and the impulse response curves are packed more and more.

The parameters listed in Table $\mathrm{V}$ are used for simulation of channel response with fading characteristics. The channel response over the coherence period is plotted in Fig 6.For the assumed parameters, Coherence time and coherence bandwidth of the channel response is $B_{c} \ll \Delta f, \quad T_{c} \quad<T_{s}$ thus the signal experiences a Fast and Frequency selective fading. Thus a closely packed spectrum in (top left) and single arrivals in many instants is seen in fig 6 .

Table IV

Channel model

\begin{tabular}{|l|l|}
\hline Parameter & value \\
\hline surface height (depth) [m] & 100 \\
\hline TX height [m] & 60 \\
\hline RX height [m] & 40 \\
\hline channel distance [m] & 5000 \\
\hline spreading factor & 1.5 \\
\hline speed of sound in water[m/s] & 1500 \\
\hline speed of sound in hard bottom [m/s] & 1200 \\
\hline Frequency & $9.2 \mathrm{KHz}$ \\
\hline Bandwidth & $10.8 \mathrm{KHz}$ \\
\hline coherence time of the small-scale variations & $69 \mathrm{~ms}$ \\
\hline Total duration of the signal & $94 \mathrm{~ms}$ \\
\hline TX vehicular angle & $0 \mathrm{rad}$ \\
\hline RX vehicular speed & $1 \mathrm{~m} / \mathrm{s}$ \\
\hline RX vehicular angle & $0 \mathrm{rad}$ \\
\hline
\end{tabular}

The transmitted frame is shown in fig7. The frame has one OFDM block of 1024 QPSK symbols, each symbol carried by one subcarrier. Each subcarrier is divided to 3samples thus the number of samples in the figure is three times the number of subcarriers. The frame gets corrupted as it passes through the fast fading channel and experiences Doppler shift proportional to wind speed and platform motion. This frame is shown in fig8. The received frame is recovered through the receiver algorithm explained in section $\mathrm{V}$ and the corresponding scatter plot is depicted in fig9. 


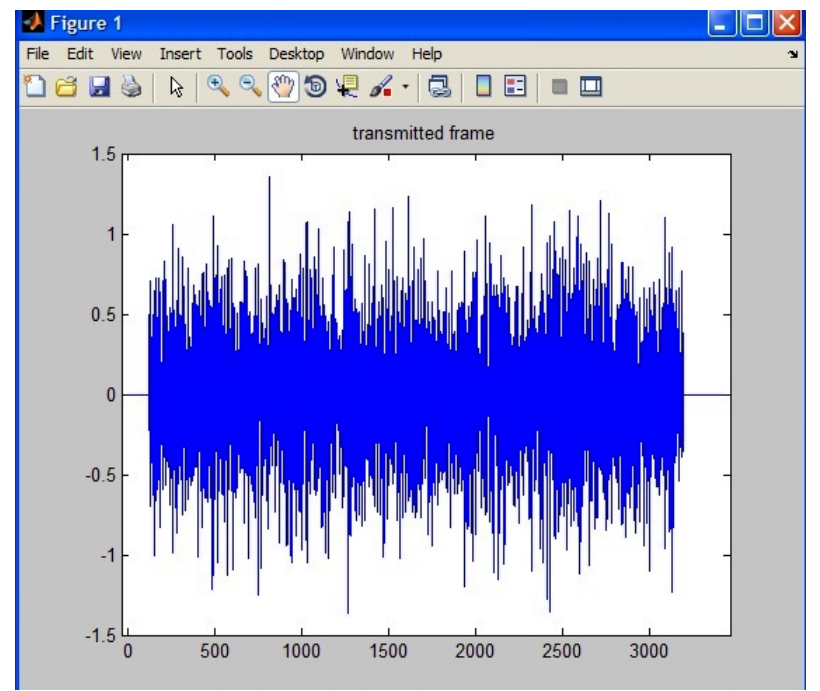

Figure 7 Transmitted OFDM frame in a packet with 1024 carriers

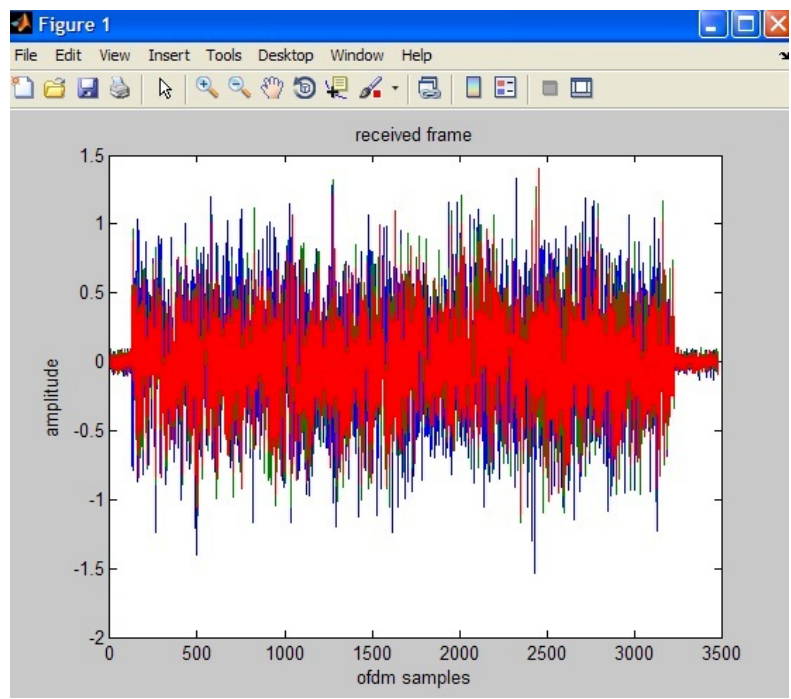

Figure 8 Received Frame with expansion on both sides corresponding to a Doppler shift of $6 \mathrm{~Hz}$

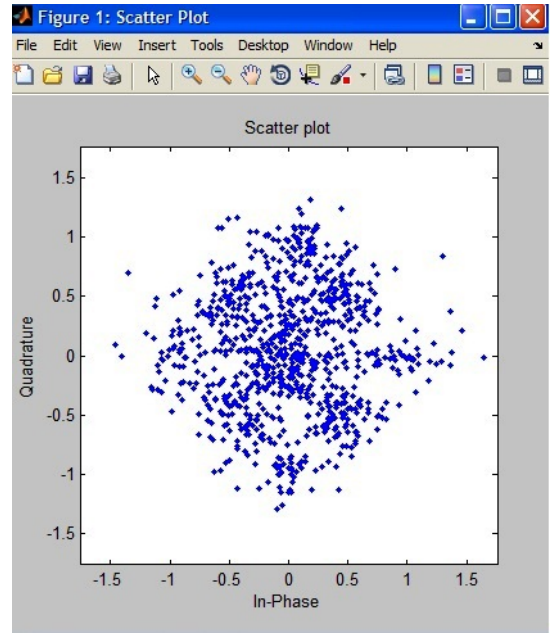

Figure 9 Scatter plot of the detected symbol

Mean square error (MSE) is calculated between the actually transmitted frame and received frame. Figure 10 shows the variation of MSE for constant channel gain and delay without equalization across blocks with different Doppler shifts. It is observed that the MSE increases as number of blocks increases. 


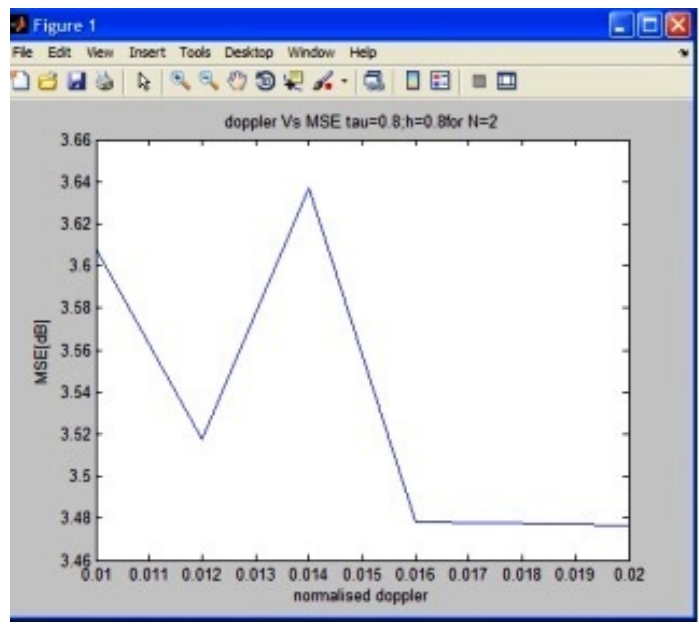

(a)

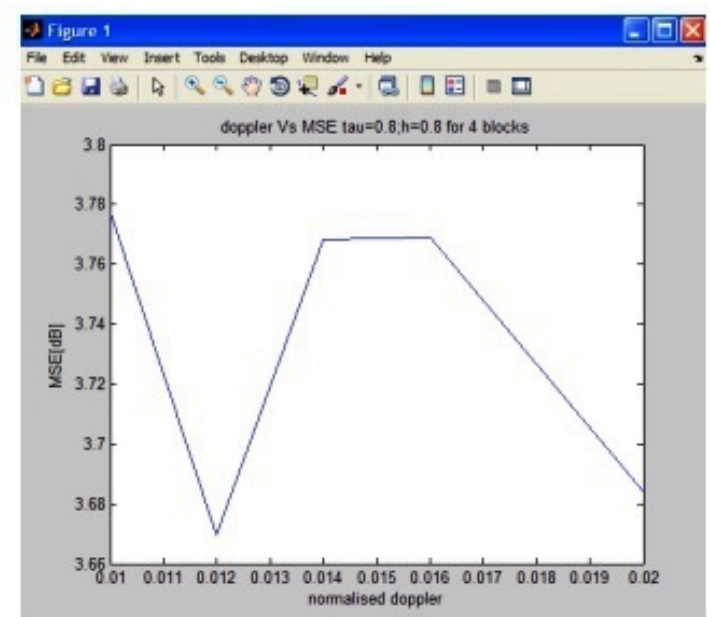

(b)

Figure 10 Variation of MSE with Doppler frequency across blocks

(a) Two blocks

(b) Four blocks

Figure11 shows the variation of MSE and bit error rate (BER) with 3 tap equalization. However the simulation results did not show any improvement. Thus there is a strong need to employ alternate equalisation techniques, Doppler compensation algorithms, spatial diversity (multiple receivers) and an effective combiner

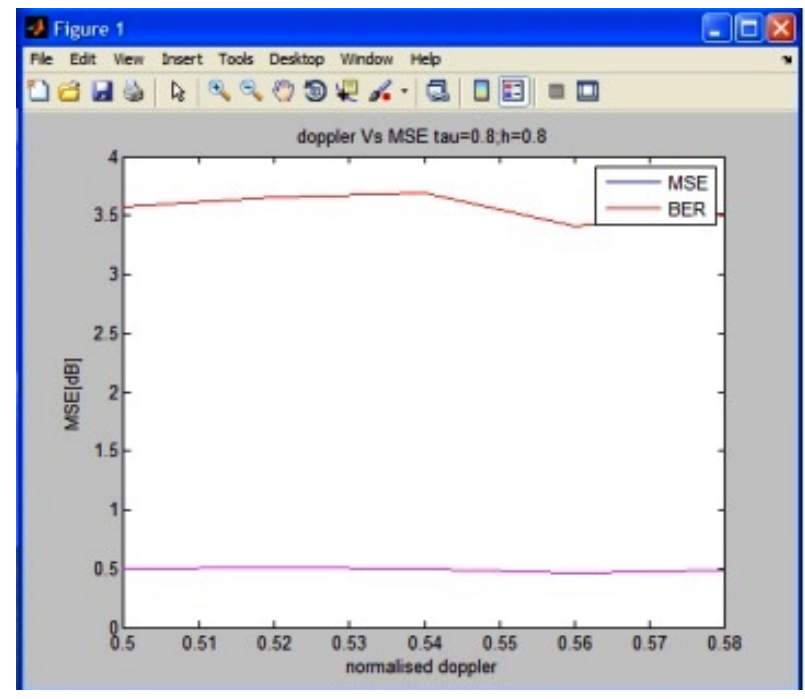

Figure 11 variation of MSE for 3 tap equalizer

\section{CONCLUSION}

In this paper, analysis of ray tracing based Doppler simulation and the effect of Doppler shift in transmission of OFDM blocks were analysed. Further work can be initialled towards the development of efficient Doppler compensation algorithms with spatial diversity (multiple receivers) and an effective combiner.

\section{REFERENCES}

[1] Srinivas Yerramalli, Urbashi Mitra,"Optimal Resampling of OFDM Signals for Multiscale Multilag Underwater Acoustic Channels," IEEE Journal of Oceanic Engineering (); Volume: 36, Issue: 1, Pages: 126-138, 2011.

[2] Xiaoka Xu, Zhaohui Wang, Shengli Zhou, and Lei Wan, "Parameterizing both path amplitude and delay variations of underwater acoustic channels for block decoding of orthogonal frequency division multiplexing," Acoustical Society of America, Pages: 46724679 April 2012.

[3] Kai Tu, Tolga M. Duman, Milica Stojanovic, and John G. Proakis, "Multiple-Resampling Receiver Design for OFDM Over DopplerDistorted Underwater Acoustic Channels," IEEE Journal of Oceanic Engineering, Vol.38, No.2, April 2013.

[4] Bayan S. Sharif, Member, IEEE, Jeff Neasham, Oliver R. Hinton, and Alan E. Adams "A Computationally Efficient Doppler Compensation System for Underwater Acoustic Communications," IEEE J. Ocean Eng., volume (25), pp. 52-61, 2000.

[5] Limin Yu, L.B. White, "Broadband Doppler compensation for rational wavelet-based UWA communication systems," proceedings of Asia-Pacific Conference on Communications, ISBN: 0-7803-9132-2; November 2005 
[6] Baosheng Li, Shengli Zhou, M. Stojanovic, L. Freitag, and P. Willett, "Multicarrier Communication over Underwater Acoustic Channels with Nonuniform Doppler Shifts," IEEE Journal of Oceanic Engineering, 2008.

[7] A.E. Abdelkareem, B. S. Sharif, C. C. Tsimenidis and J. A. Neasham, "Time varying Doppler-shift compensation for OFDM-based shallow Underwater Acoustic Communication systems," Eighth IEEE International Conference on Mobile Ad-Hoc and Sensor Systems, 2011.

[8] Gang Qiao, Wei Wang, Ran Guo, Rehan Khan and Yue Wang, "Frequency Diversity for OFDM Mobile Communication via Underwater Acoustic Channels," Journal of Marine Sci. Appl., 126-133, 2012

[9] M. Stojanovic, J. Catipovic and J.G. Proakis, "Adaptive multichannel combining and equalization for underwater acoustic communications" Journal of Acoustical Society of America. PACS numbers: 43.30, 43.60,2013.

[10] Ranjani.G, Dr.Sadashivappa.G, "Characterization of Underwater Acoustic channels", IEEE International Conference Proceedings on Applied and Theoretical Computing and Communication Technology,Oct.2015;

\section{AUTHOR PROFILE}

Author1: Ranjani G has obtained M.Tech from VTU, Belgaum. She has 14 Years of teaching experience. She has published technical papers in many international conferences and journals in the field of signal processing and wireless communication. She is currently Assistant professor at Telecommunication Engineering Department, RVCE, Bangalore. Her area of interest are Engineering education practices, Signal processing \& applications

Author2: Dr. G. Sadashivappa, has obtained his doctoral degree from VTU, Belgaum. He has 28 Years of teaching experience and 6 years of research experience. He has authored two text books .He has chaired many international conferences. He was the Principle Investigator for the project "On Study and development of Image Compression Algorithms for Satellite Images" supported by ISRO. He is currently professor at Telecommunication Engineering Department, RVCE, Bangalore. His area of interest are Fibre Optic communication, Signal processing \& applications 